\title{
GEOMETRÍA COMPUTACIONAL: EL PROBLEMA DEL CERCO CONVEXO
}

\section{RESUMEN}

El artículo tiene por objetivo presentar los conceptos fundamentales de la geometría computacional y mostrar una solución al problema del cerco convexo, utilizando el algoritmo de Graham. Para la implementación de la solución, los autores usan el lenguaje de programación Java.

Palabras Clave: Geometría computacional, diagramas de Voronoi, planeamiento de movimiento, problema del cerco convexo, algoritmo de Graham.

COMPUTATIONAL GEOMETRY: THE CONVEX HULL PROBLEM

ABSTRACT

The objective of this work is to show fundamental concepts of computational geometry and one solution for the convex hull problem, using the Graham algorithm. The authors use the Java language programming in order to implement the solution.

Key words: Computational geometry, Voronoi diagrams, motion planning, convex hull problem, Graham algorithm.

(1) Ingeniero Industrial. Profesor del Departamento de Ingeniería de Sistemas e Informática, UNMSM. E-mail: eruizl@unmsm.edu.pe

(2) Ingeniero Industrial. Profesor del Departamento de Ingeniería de Sistemas e Informática, UNMSM. E-mail: eraffo@@unmsm.edu.pe

\section{INTRODUCCIÓN}

La geometría computacional es una rama de la ciencia de la computación y emergió en la década de 1970. Estudia los algoritmos que resuelven problemas geométricos. Su aplicación, va desde el diseño de los VLSI, hasta el CAD (Computer-Aided Design), el CAM, computación gráfica y GIS (sistemas de información geográfica)[1].

Imagine los teléfonos públicos en una urbanización y como contar con un mapa que muestre las regiones en donde se encuentre el teléfono más cercano. Una distribución posible se presenta en la Figura 1.

A la subdivisión de un área o localidad en regiones, se conoce como diagrama de Voronoi; y para hacer esto se hace uso de algoritmos geométricos.

Sea un conjunto de obstáculos geométricos (como los mostrados en la Figura 2), y asuma que se desea encontrar la conexión más corta entre dos puntos geométricos, salvando obstáculos. Este problema que es crucial en robótica se conoce como planeamiento del movimiento (motion planning), y el cual hace uso de algoritmos geométricos.

Un clásico problema en CG (del ingles Computational Geometry) es el cerco convexo o $\mathrm{CH}$ (Convex Hull); este crea un subconjunto $S$ de un plano denominado convexo si y solo sí, cualquier par de puntos $p, q \in S$; es decir el segmento pq está contenido completamente en $S$ (ver Figura 3).

El Cerco convexo $\mathrm{CH}(S)$ (se lee $\mathrm{CH}$ de $S$ ) es el más pequeño conjunto convexo en $S$.

Veamos el siguiente ejemplo:

entrada : $p_{1}, p_{2}, p_{3}, p_{4}, p_{5}, p_{6}$

salida $: p_{4}, p_{5}, p_{6}, p_{2} \quad$ (ver Figura 4$)$

\section{MÉTODOS GEOMÉTRICOS ELEMENTALES}

Las aplicaciones en geometría computacional actúan sobre objetos geométricos simples: punto, línea y polígono [2].

Puntos. Es el objeto fundamental, y es considerado como un par de enteros: las coordenadas habituales en el plano cartesiano. 


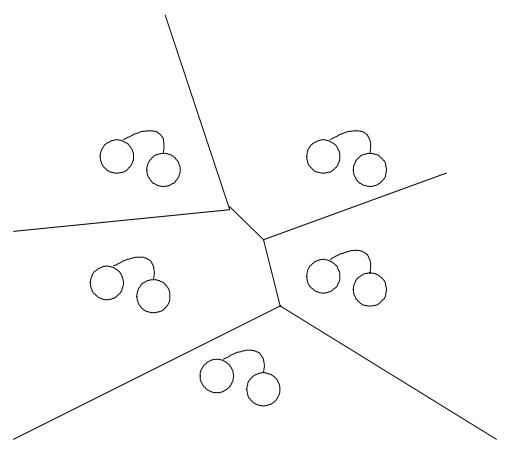

Figura 1. Área dividida en regiones

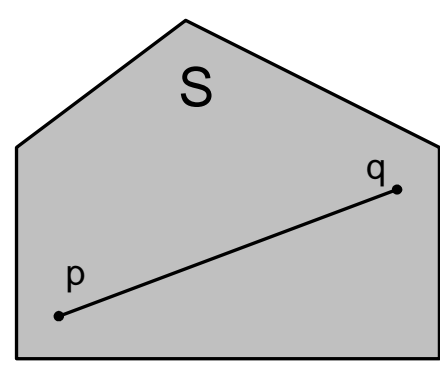

Figura 3. El problema del cerco convexo

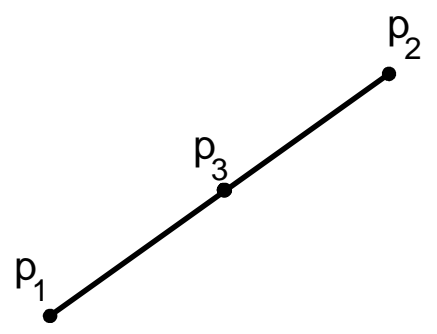

Figura 5. Unalínea
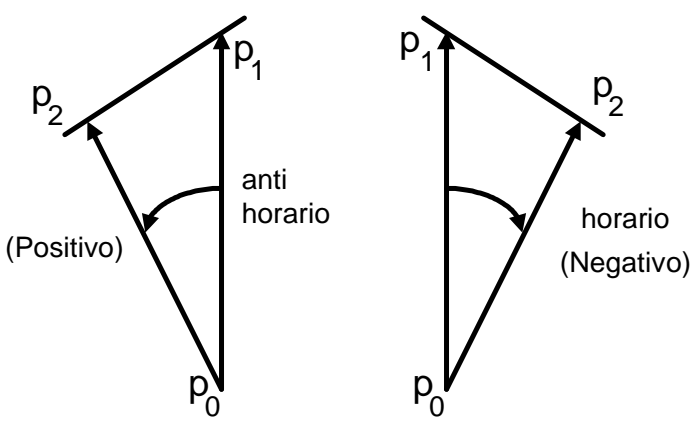

Figura 7. Sentido horario $p_{1} \times p_{2}$

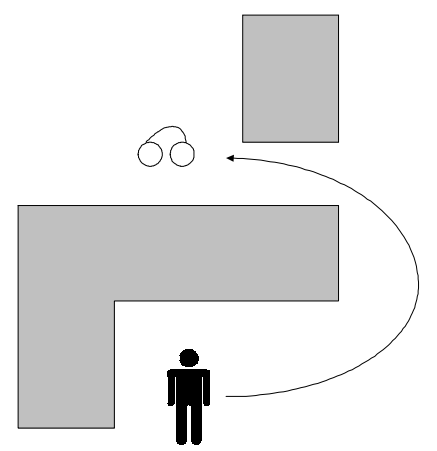

Figura 2. Esquema del planeamiento del movimiento

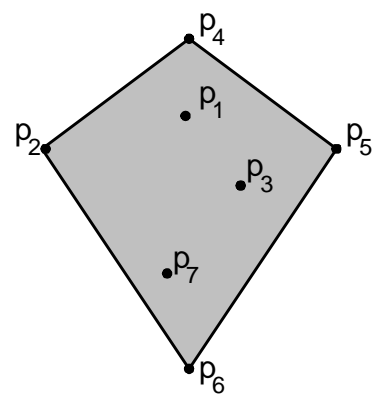

Figura 4. Un cerco convexo

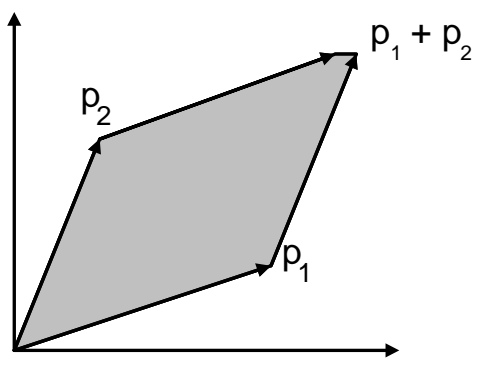

Figura 6 . Vectores y el producto cruz

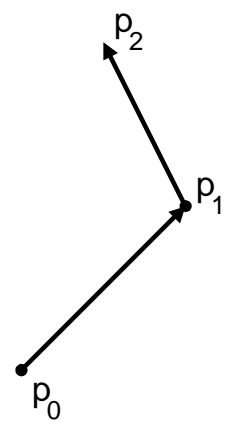

Figura 8. Producto cruzado negativo 
Línea. Una línea es un par de puntos, que se supone están unidos por un segmento de línea recta.

Para especificar las propiedades de un segmento de línea recta, se define la combinación convexa de $p_{1}=$ $\left(\mathrm{x}_{1}, \mathrm{y}_{1}\right)$ y $p_{2}=\left(\mathrm{x}_{2}, \mathrm{y}_{2}\right)$, como un punto $p_{3}=\left(\mathrm{x}_{3}, \mathrm{y}_{3}\right)$; existiendo un $\alpha$, tal que $0 \leq \alpha \leq 1$, y $p_{3}=\alpha p_{1}+(1-$ $\alpha) p_{2}$, siendo $p_{3}$ un punto que está contenido en el segmento de línea $p_{1} p_{2}$ (ver figura 5).

$\mathrm{x}_{3}=\alpha \mathrm{x}_{1}+(1-\alpha) \mathrm{x}_{2}$

$y_{3}=\alpha y_{1}+(1-\alpha) y_{2}$

Considere los vectores $p_{1}$ y $p_{2}$; su producto cruzado $p_{1} \times p_{2}$ se interpreta como el área del paralelogramo formado por $(0,0), p_{1}, p_{2}$ y $p_{1}+p_{2}$ (ver figura 6 ); y se define como:

$$
\begin{aligned}
p_{1} x p_{2} & =\operatorname{det}\left(\begin{array}{ll}
x_{1} & x_{2} \\
y_{1} & y_{2}
\end{array}\right) \\
& =\mathrm{x}_{1} y_{2}-x_{2} y_{1}
\end{aligned}
$$

Si $p_{1}$ y $p_{2}$ es positivo, entonces $p_{1}$ es sentido horario de $p_{2}$ en caso que el producto cruzado sea cero, se dice que son colineales.

El problema de determinar si dos segmentos consecutivos se encuentran a la izquierda o a la derecha: $p_{0} p_{1}$ y $p_{1} p_{2}$ con respecto a $p_{1}$; equivale a encontrar el ángulo entre $p_{0} p_{1} p_{2}$. Utilizando el producto cruzado se tiene que

$$
\left(\mathrm{p}_{2}-\mathrm{p}_{0}\right) \times\left(\mathrm{p}_{1}-\mathrm{p}_{0}\right)
$$

En caso que el producto cruzado sea negativo $p_{0} p_{2}$ es antihorario con respecto a $p_{0} p_{1}$ y se encuentra a la izquierda $p_{2}$. Un producto cruzado positivo es tomado como sentido horario con respecto a $p_{0} p_{1} y$ $\mathrm{p}_{2}$ es a la derecha. Al respecto ver la Figura 8.

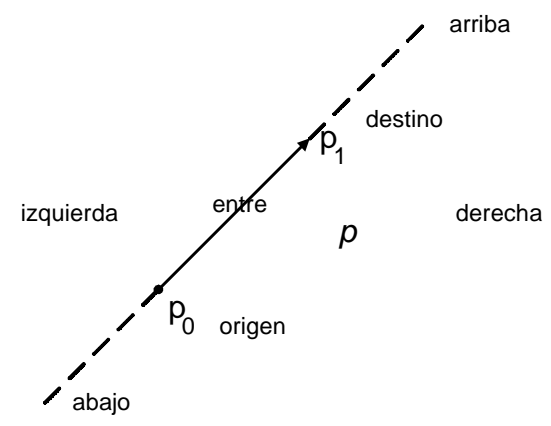

Figura 9. Clasificación de $P$
Se puede clasificar el punto $P$ con respecto a una región.

Así puede un punto $P$, encontrarse: a la izquierda, derecha, origen, destino, entre los dos puntos, arriba o abajo (véase la Figura 9).

\section{SEGMENTOS DE LÍNEA}

Un segmento de línea $\overline{p_{0} p_{1}}$, consiste de los puntos finales $p_{0}$ y $p_{1}$ (véase Figura 10).

Si $p_{o}$ es el origen de un segmento de línea y $p_{1}$ es el destino, entonces es un segmento de línea dirigido $p_{0} p_{1}$; denominado arco o borde (edge). Un arco posee dos puntos finales: origen y destino; por defecto el punto $p_{0}$ es $(0,0)$ y destino es $(1,0)$.

La rotación de un arco es mover a $90^{\circ}$ en sentido horario los puntos finales (véase la Figura 11).

donde:

$m=0.5^{*}\left(p_{1}-p_{0}\right)$

$p=p_{1}-p_{0}$

y como

$$
\begin{aligned}
& n \cdot\left(p_{1}-p_{0}\right)=0, \text { con } p_{1}-p_{0}=(x, y) \\
& n=p(y,-x) \\
& \text { origen }=m-0.5^{\star} n \\
& \text { destino }=m+0.5^{\star} n
\end{aligned}
$$

Se observa que una doble notación o twice es la rotación de una rotación (Ver figura 12)

Para determinar el punto en $\mathrm{t}$, se tiene que este es igual a $p_{0}+t *\left(p_{1}-p_{0}\right)$

Para ínter seccionar un objeto arco con otro denominado $f$, se tiene que:

$\mathrm{P}(\mathrm{t})$ - $\mathrm{C}$ coincide con $\overline{c d}$ n. $(\mathrm{P}(\mathrm{t})-\mathrm{C})=0$

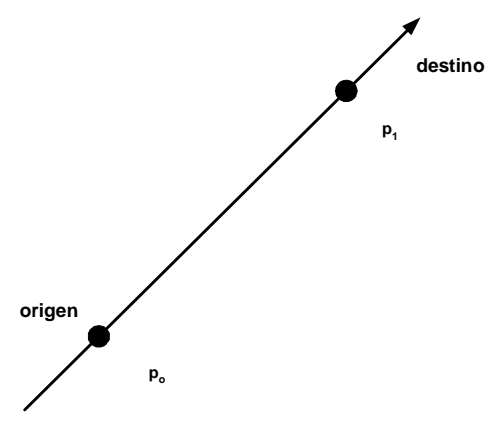

Figura 10. Segmento de línea $\overline{p_{0} p_{1}}$ 


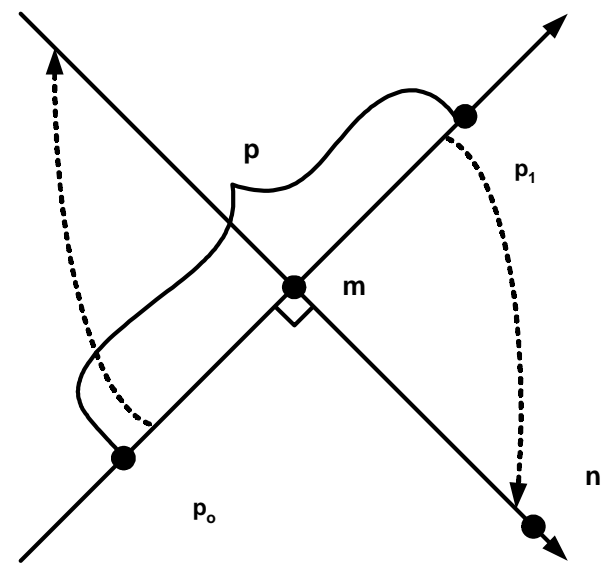

Figura 11. Rotación o arco

$n \cdot((a+t(b-a))-c)=0$

$n \cdot(a-c)+\operatorname{tn} .(b-a)=0$

$$
t=-\frac{n \cdot(a-c)}{n \cdot(b-a)}
$$

Si n.(b-a) es cero; se tiene que b-a y d-c son perpendiculares al vector $n$. Al clasificar el arco $f$, si este es LEFT o RIGHT retorna PARALELO; en otro caso COLINEAL.

El valor de tes $\frac{n \cdot(a-c)}{n \cdot(b-a)}$

que indica que hay intersección, retornando INTERSECCION (véase la Figura 13).

Para evaluar la pendiente (o slope, en inglés), la con-

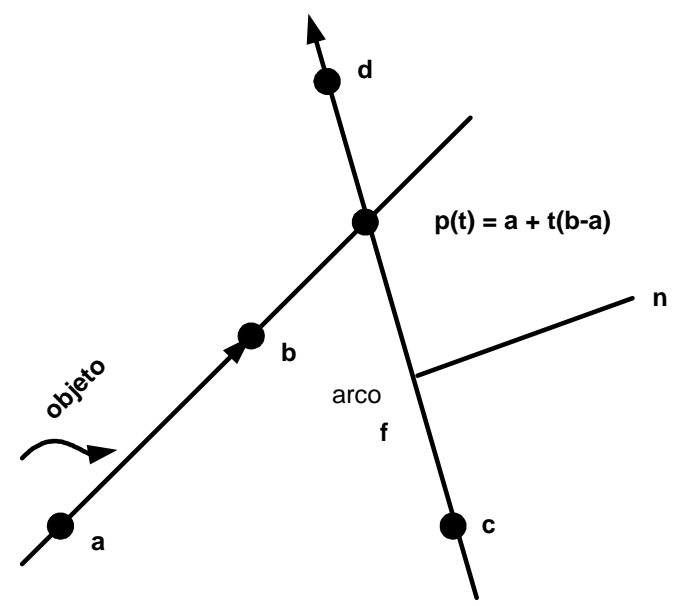

Figura 13. Intersección

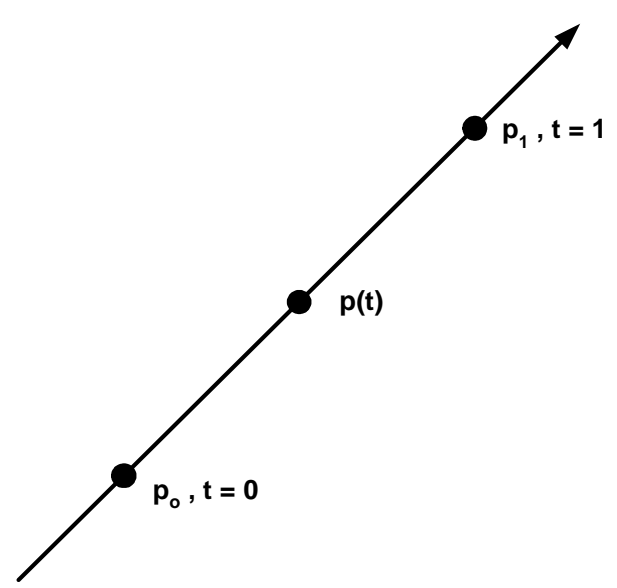

Figura 12. Rotación de rotación

dición es $p_{0} \cdot x$ sea diferente a $p_{1} \cdot x$ (porque en otro caso es $\square$ ):

$$
\text { pendiente }=\frac{p_{1} . y-p_{0 . y}}{p_{1 . x}-p_{0 . x}}
$$

El valor de la ordenada $y$ en base a la abscisa $x$ (según la figura 14), es:

$$
y=\text { pendiente }^{*}\left(x-p_{0 . x}\right)+p_{0 . y}
$$

\section{EL PROBLEMA DEL CERCO CONVEXO}

Un polígono convexo[5], es un polígono con la propiedad de que cualquier segmento de recta cuyos extremos estén en el polígono cae por completo dentro

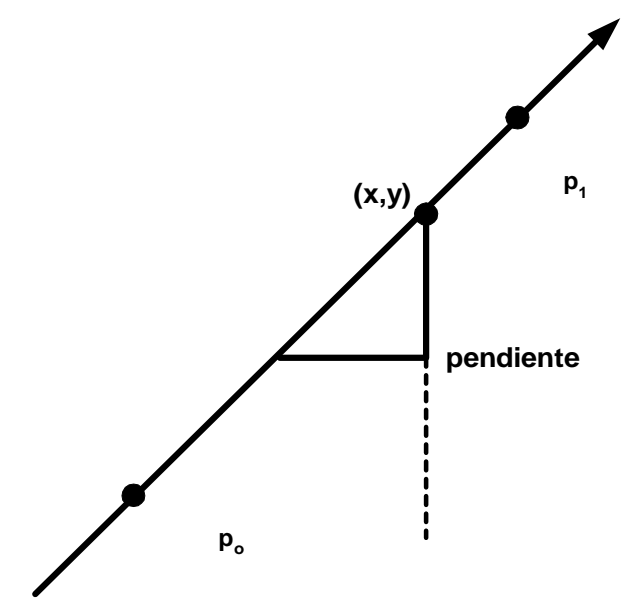

Figura 14 . El concepto de pendiente de una recta 


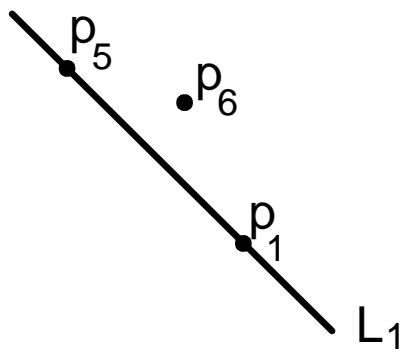

Figura 15. $\mathrm{P}_{6}$ no forma parte del cerco

del polígono. El problema del cerco convexo consiste en encontrar el área más pequeña de un polígono convexo que encierre un conjunto de puntos en el plano. Como se vio anteriormente, las figuras 3 y 4 presentan un cerco convexo, así también la Figura 16.

Sea un conjunto $S$ en el plano; y $P$ un punto en $S$. Se dice que $P$ es un punto del cerco, si existe una línea $\mathrm{L}$ a través de $P$, tal que todos los puntos en $\mathrm{S}$ excepto $P$, se encuentran a un lado de $L$ (excepto $P$, ninguno pertenece a la línea).

En la Figura $15, p_{6}$ no es un punto del cerco por encontrarse a un lado de $L_{1}$.

El cerco convexo de un conjunto finito de puntos $S$, consiste de puntos del cerco ordenados que se encuentran en el borde de $S$. Para la Figura 16 el cerco convexo es la secuencia de los puntos $p_{1}, p_{2}$, $p_{3}, p_{4}, p_{5}$.

El cerco convexo de un conjunto finito de puntos $S$ en el plano, es la secuencia $p_{1}, p_{2}, \ldots, p_{n}$ de puntos de cercos de $S$ en el siguiente orden:

1. El punto $p_{1}$ es el punto con la coordenada mínima $y$ ( $p_{1}$ es un punto de cerco).

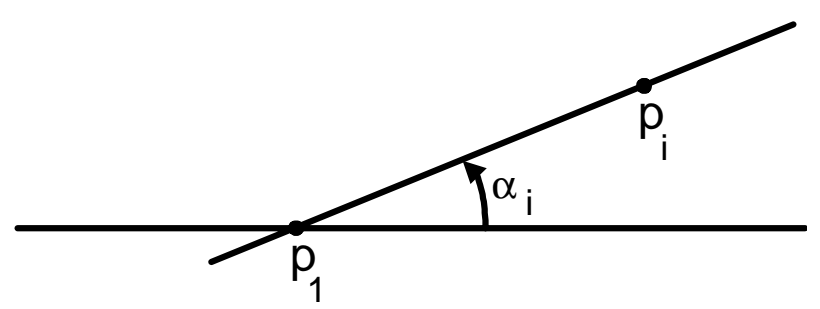

Figura $17 . \mathrm{P}_{6}$ no forma parte del cerco

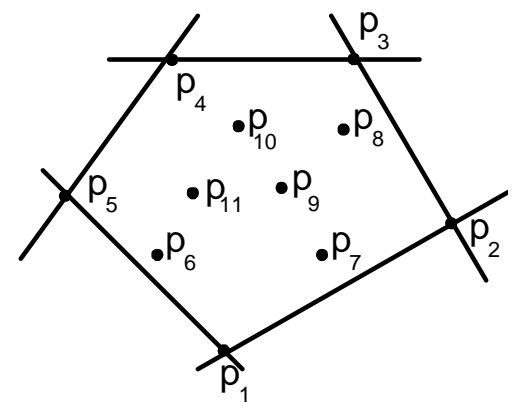

Figura 16. Un cerco convexo

2. Para $\mathrm{i} \geq 2$, sea $\alpha_{i}$ el ángulo desde la horizontal al segmento de línea $p_{1}, p_{\mathrm{i}}$. Los puntos $p_{2}, p_{3}, \ldots, p_{\mathrm{n}}$ están ordenados tal que $\alpha_{i}, \alpha_{i}, \ldots, \alpha_{n}$ están en una secuencia ordenada. (Ver figura 17)

En la Figura 18; $p_{1}$ tiene la mínima coordenada $y$; entonces este es el primer punto listado en el cerco convexo. Como se muestra, los ángulos desde la horizontal a las líneas segmentos $p_{1}, p_{2} ; p_{1}, p_{3} ; p_{1}$, $p_{4} ; p_{1}, p_{5}$ crecen.

\section{ALGORITMO DE GRAHAM}

En el algoritmo de Graham (R.L. Graham, An efficient algorithm for determining the convex hull of a finite planar set, Information Processing letters 1, 132-133; 1972) los puntos son ordenados de acuerdo al ángulo tomando un punto inicial. La secuencia o pasos del algoritmo se presentan a continuación.

a. Primero encontrar el punto $p_{1}$ con mínima coordenada y; en el caso que varios puntos tengan el mismo valor, tomar el del mínimo valor de $x$.

b. Ordenar todos puntos de $P$ de $S$ de acuerdo al ángulo desde la horizontal al segmento de línea $p_{1}, p$.

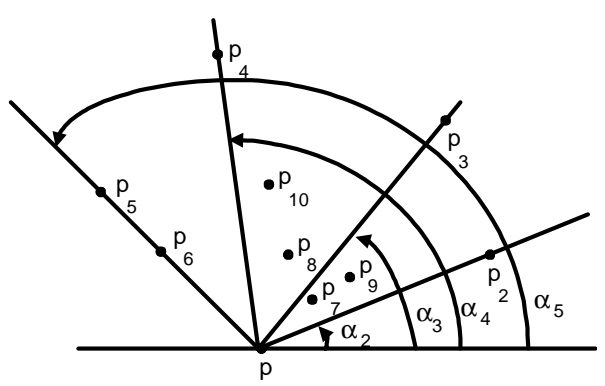

Figura 18. Un cerco convexo 


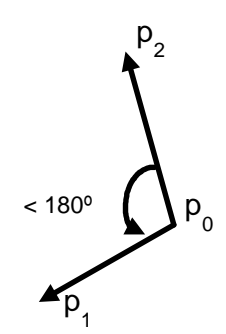

izquierda

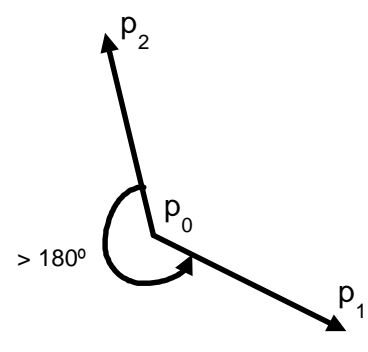

derecha
Figura 19. Comparación de ángulos

c. Finalmente examinar los puntos en orden y descartar aquellos que no están en el cerco convexo.

Para implementar la estrategia usada en el algoritmo, se desarrolla un camino para comparar ángulos.

El producto cruzado determina cuando un punto no se encuentra en cerco convexo y deberá ser descartado.

Suponga que visita $p_{1}, p_{0}$ y $p_{2}$ en ese orden y los segmentos de línea $p_{0}, p_{2}$ y $p_{0}, p_{1}$ tienen ángulos menores que $180^{\circ}$ entonces $p_{0}$ se mueve a la izquierda, en otro caso se mueve a la derecha.

El producto cruz de $p_{0}, p_{1}, p_{2}$ es:

$\operatorname{cross}\left(p_{0}, p_{1}, p_{2}\right)=\left(\mathrm{y}_{2}-\mathrm{y}_{0}\right)\left(\mathrm{x}_{1}-\mathrm{x}_{0}\right)-\left(\mathrm{y}_{1}-\mathrm{y}_{0}\right)\left(\mathrm{x}_{2}-\mathrm{x}_{0}\right)$

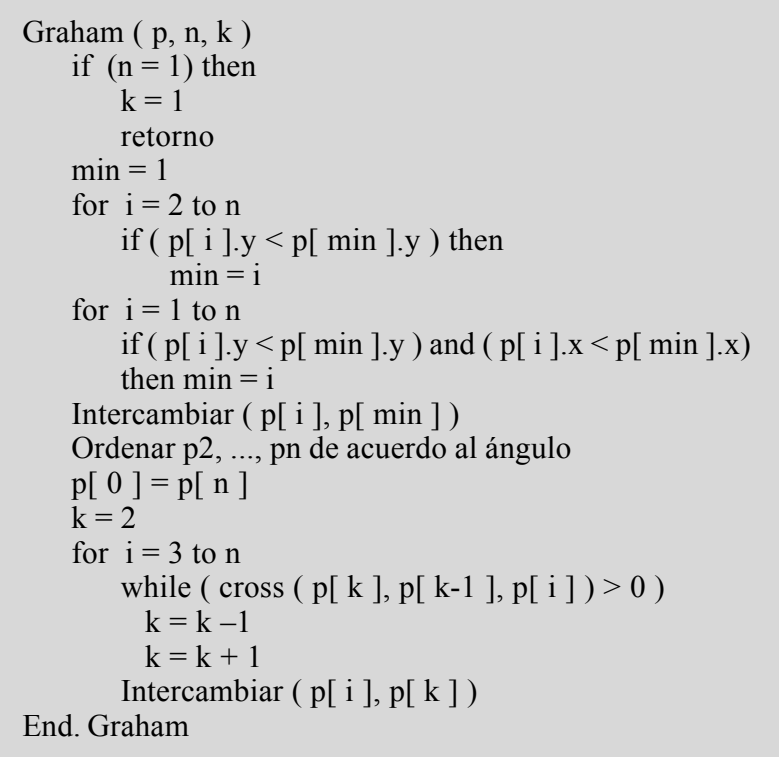

Figura 21 . Seudo código para el algoritmo de Graham

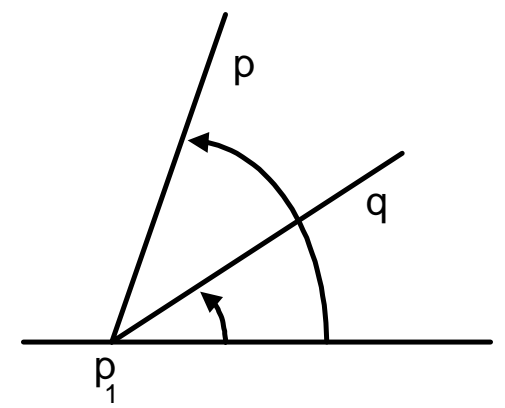

Figura 20. Comparando: $p>q$

esto, implica que se mueve a la izquierda si cross $\left(p_{0}, p_{1}, p_{2}\right)<0$.

De igual manera $p_{0}, p_{1}, p_{2}$ se mueve a la derecha si cross $\left(p_{0}, p_{1}, p_{2}\right)>0$. Cuando cross $\left(p_{0}, p_{1}, p_{2}\right)=0$, entonces los puntos $p_{0}, p_{1}, p_{2}$ son colineales.

El algoritmo Graham toma sistemáticamente los puntos $p \neq q$ en el ordenamiento y compara $p$ y $q$. Si el producto cross $\left(p_{1}, p, q\right)$ es $<0$ entonces $p, p_{1}, q$ se mueven a la izquierda; es decir $p>q$. Al respecto examine el seudo código de la Figura 21.

Sea el conjunto $S$ ordenado de acuerdo a sus ángulos (véase Figura 22). El algoritmo examina $p_{1}, p_{3}, p_{4}$, moviéndose hacia la izquierda, luego $p_{2}$ es retenido. Después se examina $p_{2}, p_{3}, p_{4}$, reteniéndose $p_{3}$ (ver Figura 23).

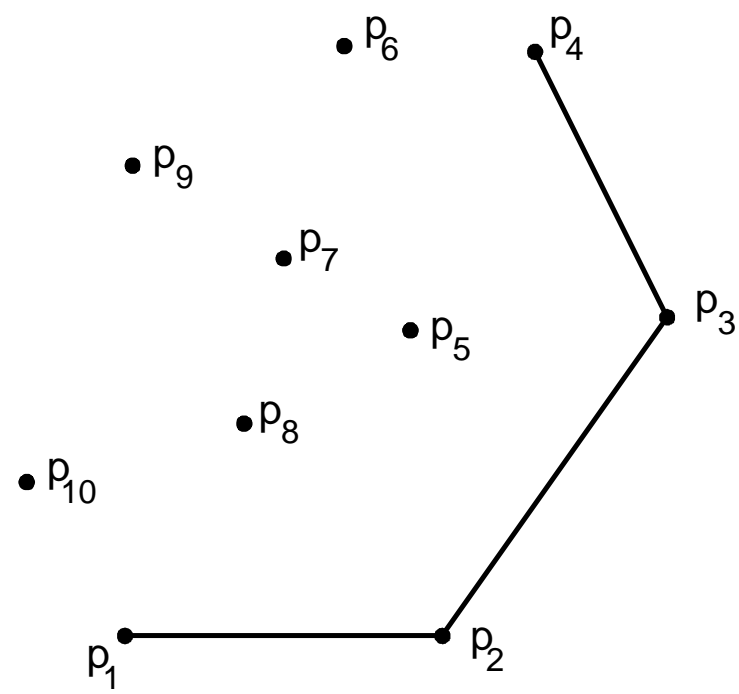

Figura 22. Conjunto $S$ de puntos 


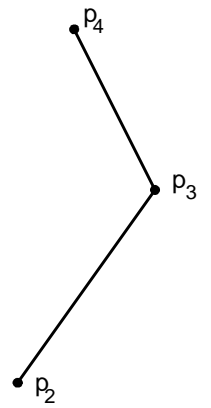

Figura 23. Encontrando $p_{2}, p_{3}, p_{4}$

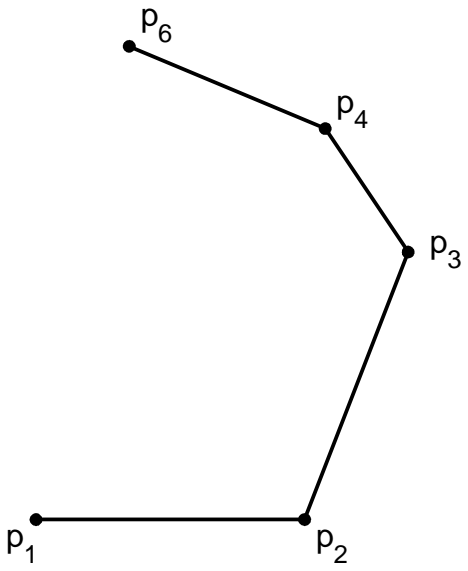

Figura 25. Obteniendo $p_{6}$

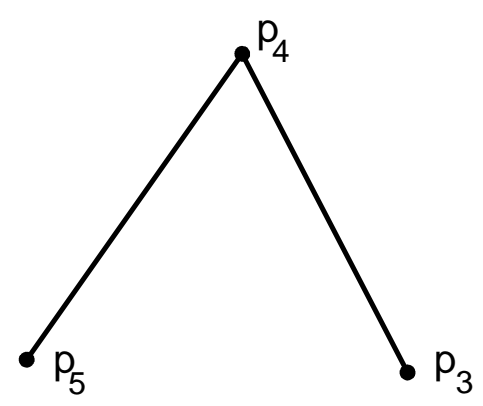

Figura 24. Examinando $p_{3}, p_{4}, p_{5}$

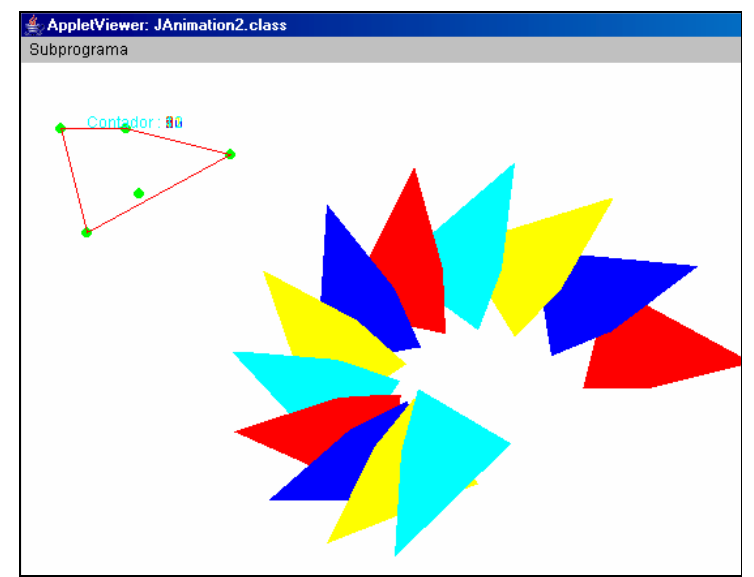

Figura 26. Salida para Graham.java

public void add(Point2D p)

* Fecha :1/05/2004

$* * * 1$

import Point2D;

public class Graham \{ private int $\mathrm{n}$;

private int $\mathrm{k}$;

private Point2D puntos[];// puntos en el conjunto $\mathrm{S}$ public static Point2D[] hull;

public Graham(int m)

\{

puntos=new Point2D $[\mathrm{m}+1]$;

puntos $[\mathrm{m}]=$ new Point $2 \mathrm{D}()$;

\}

public Graham()

\{

this(10);// por defecto if( $\mathrm{n}<$ puntos.length-1) puntos $[\mathrm{n}++]=\mathrm{p}$;

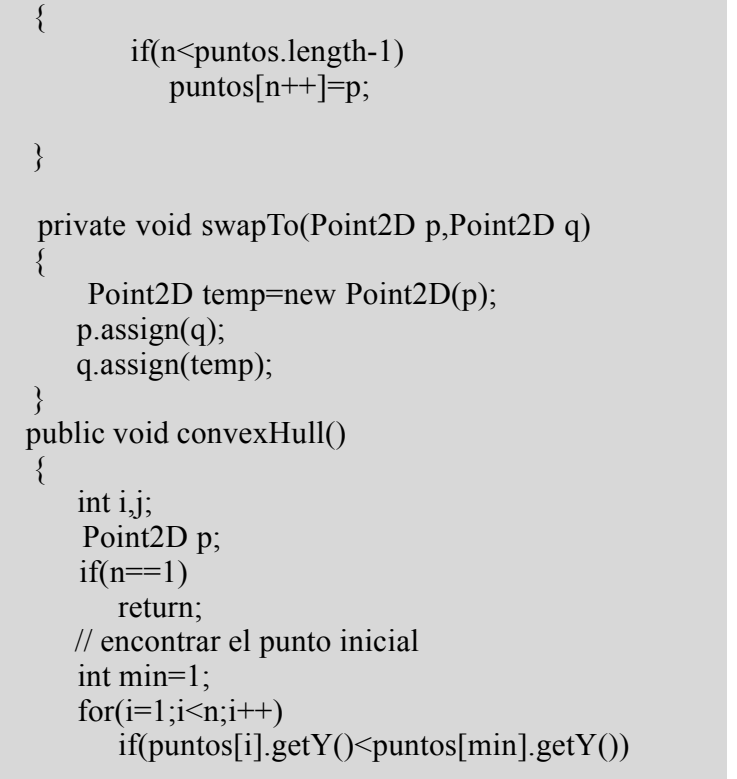



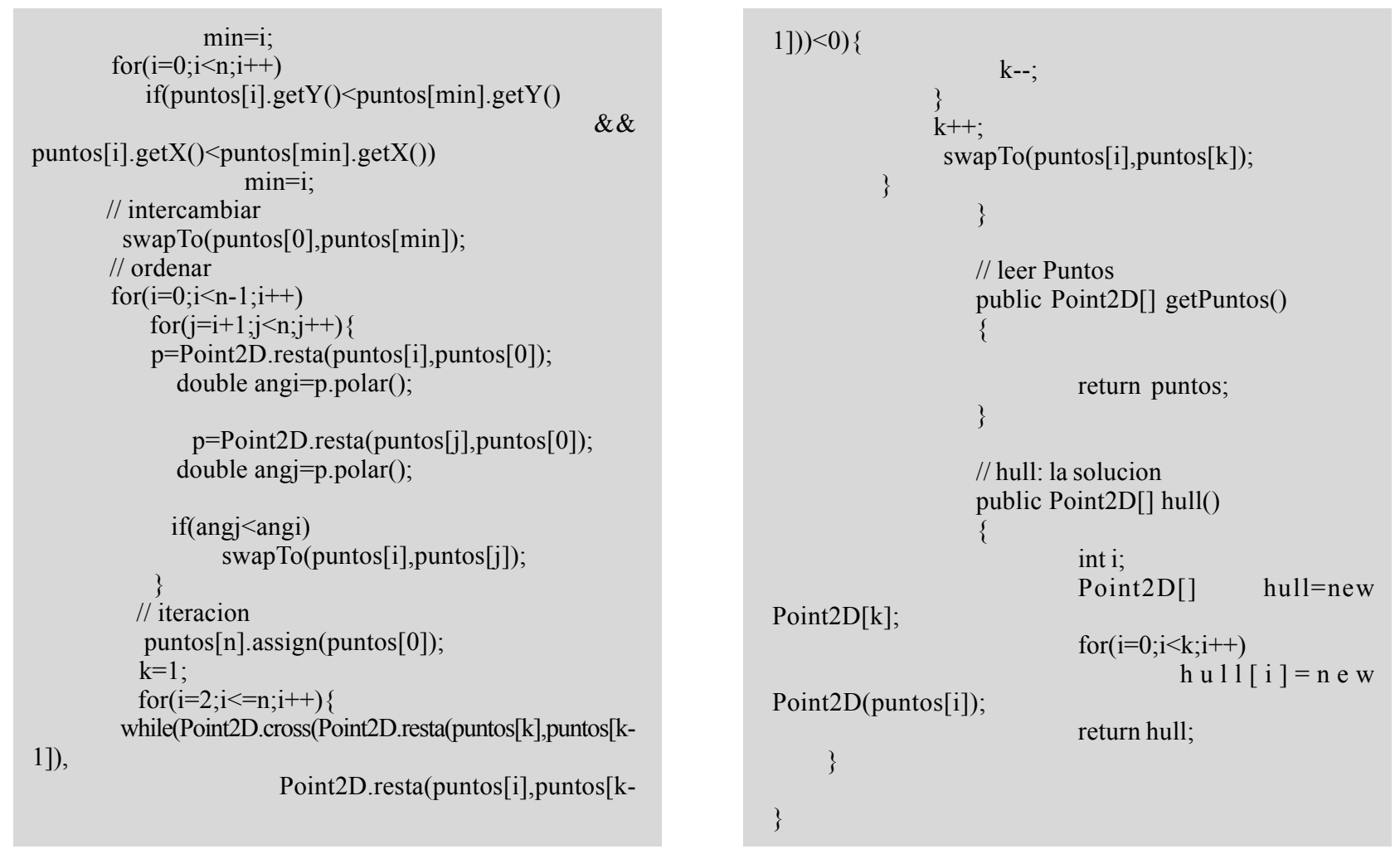

Figura 276. Código del archivo7 Graham.java

En la siguiente iteración examina $p_{3}, p_{4}, p_{5}$ y se retiene $p_{4}$ (ver figura 24).

Al examinar $p_{4}, p_{5}, p_{6}$ se descarta $p_{5}$ y se regresa a $p_{3}, p_{4}, p_{6}$, se retiene $p_{4}$.

Al examinar $p_{4}, p_{6}, p_{7}$ se retiene $p_{6}$. En la siguiente se examinan $p_{6}, p_{7}, p_{8}$ se retiene $p_{7}$. En $p_{7}, p_{8}, p_{9}$ se descarta $p_{8}$. El algoritmo regresa a $p_{6}, p_{7}, p_{9}$ y se descarta $p_{7}$. El algoritmo regresa a $p_{4}, p_{6}, p_{9}$ y se retiene $p_{6}$.

A continuación se presenta el código JAVA para el archivo Graham.java (Figura 27: a y b). La animación desde un applet, se presenta en la Figura 26. Los archivos: Point2D.java, JgrahamShow.java, JgrahamShow.html, se encuentran en [3].

\section{CONCLUSIONES}

El artículo presenta los conceptos fundamentales de la geometría computacional y una solución a uno de los problemas clásicos en este campo: el cerco convexo.

La solución es implementada en un applet de Java, el mismo que aprovecha la interfase grafica para presentar una animación de un polígono (cerco convexo) en la salida estándar.

\section{BIBLIOGRAFÍA}

1. Laszlo, M. J. (1996). Computational Geometry and Computer Graphics in C++. Prentice-Hall, Inc, U.S.A.

2. Preparata, F. P.; Michael Ian Shanos. (1988). Computational Geometry An Introduction. SpringerVerlag. U.S.A.

3. Raffo Lecca, E. (2005). Curso: Mi Primer Java. En: http://industrial.unmsm.edu.pe/org/apya/daisi/ eraffol/website/index.htm

4. Cormen, Thomas; Leiserson, Charles; Rivest, Ronald, and Stein, Clifford. (2001). Introduction To Algorithms. Second Edition. McGraw-Hill Book Company and MIT Press. U.S.A.

5. Weiss, Mark A. (2002). Data Structures and Problem Solving Using Java. Second Edition. Adisson-Wesley. U.S.A. 